\title{
Self-organization and nanostructure formation in chemical vapor deposition
}

\author{
Daniel Walgraef* \\ Instituto de Física Interdisciplinar y Sistemas Complejos (CSIC-UIB), E-07122 Palma de Mallorca, Spain
}

(Received 19 July 2013; published 10 October 2013)

\begin{abstract}
When thin films are grown on a substrate by chemical vapor deposition, the evolution of the first deposited layers may be described, on mesoscopic scales, by dynamical models of the reaction-diffusion type. For monatomic layers, such models describe the evolution of atomic coverage due to the combined effect of reaction terms representing adsorption-desorption and chemical processes and nonlinear diffusion terms that are of the Cahn-Hilliard type. This combination may lead, below a critical temperature, to the instability of uniform deposited layers. This instability triggers the formation of nanostructures corresponding to regular spatial variations of substrate coverage. Patterns wavelengths and symmetries are selected by dynamical variables and not by variational arguments. According to the balance between reaction- and diffusion-induced nonlinearities, a succession of nanostructures including hexagonal arrays of dots, stripes, and localized structures of various types may be obtained. These structures may initiate different growth mechanisms, including Volmer-Weber and Frank-Van der Merwe types of growth. The relevance of this approach to the study of deposited layers of different species is discussed.
\end{abstract}

DOI: 10.1103/PhysRevE.88.042405

PACS number(s): 81.15.Aa, 68.55.-a, 62.23.St, 81.15.Gh

\section{INTRODUCTION}

Overwhelming progress has recently been made in the area of nanoscale science and technology. For example, the formation of self-assembled and self-organized nanostructures in deposited layers on solid surfaces has become the subject of intense research activity due to its fundamental and technological relevance. Due to the ever-growing technological importance of on-demand tailored nanomaterials, it is of capital economic and scientific interest to understand and master their formation and growth since growth mechanisms usually determine most of their properties and textures. Furthermore, the ability to probe structure-property relationships on an appropriate length scale is a primary driver of progress in nanotechnology. As a result, the modeling of nanostructure formation and properties is one of the most active research areas in materials science [1].

For thin-film growth, for example, numerous computer simulation methods have been developed since the 1970s. These investigations revealed detailed information on growing films, such as island shapes, step formation, and surface roughening [2,3]. Unfortunately, in molecular dynamics and Monte Carlo approaches, the computational time required to simulate thin-film growth under realistic deposition rates is often excessive. Effectively, a significant constraint of these methods is that, due to their atomistic nature, they can deal only with small systems, with linear dimension under a micron. Other approaches, based on discrete lattices, are able to simulate thin-film growth under realistic deposition rates even in three dimensions. Nevertheless, attempts to bridge molecular dynamics and Monte Carlo method succeeded in simulating small polycrystalline films [4]. This is why continuum approaches are particularly interesting, since they are capable of simulating growth of thin films of larger dimensions [5]. In these methods, the film surface is represented by a series of mesh points that move according to material flux exchanged between neighboring regions. The drawback of

*dwalgraef@ifisc.uib-csic.es continuum simulations is that they provide information about the film surface only. Textures and microstructures are thus ignored in these descriptions. In contrast, continuous models, based on rate equations of the partial differential equation type, have also been proposed to describe mesoscopic scales, with the hope to improve the understanding of deposition processes and film surface evolution at scales inaccessible by both traditional equipment (macroscopic) and feature scales (microscopic) models [6].

Up to now, these models have had limited predictive capability because of a rough description of kinetic processes such as atomic diffusion or deposition. Nevertheless, such capability could be greatly enhanced in the framework of multiscale modeling. Effectively, the concept of multiscale material modeling has been developed to bridge the gap between atomistic and continuum methods and to link them in a consistent way $[7,8]$. The aim is to obtain a reliable description of material behavior, from microscopic to macroscopic scales. This program should be realized by coupling models for different length scales. The results from smaller scales are fed to larger scales, with appropriate mesh redefinition, and the results from larger scales are being fed back to the smaller ones, in a back and forth process hopefully ending in a quantitatively reliable solution. In the case of thin-film growth, if information from each scale is transferred correctly to the other scales, one would expect to be able to follow the evolution of film textures, surface topography, the effect of microstructures on local deposition rates, etc. In this framework, the interest of mesoscopic continuous models would be to link microand macroscales and provide not only qualitative but also quantitative descriptions of thin-film growth and nanostructure formation.

In this framework, continuous mesoscopic models have already been proposed to describe the spontaneous ordering of nanostructures or quantum dots in multicomponent epilayers on a substrate. In some of them, ordering result from the balance between coarsening effects induced by the spinodal decomposition of the solid solution that forms the film and refining effects induced by concentration-dependent surface 
stresses [9-12]. Other models describe surface growth through phenomenological evolution equations, of the gradient type, similar to the Cahn-Hilliard equation, and coupled with atomic attachment kinetics, wetting or de-wetting, and stress effects to mimic atomic deposition and film-substrate interactions, driven by substrate elasticity, wetting stress, or misfit strains [13-18]. They describe the formation of quantum dots and their self-organization in regular patterns. These patterns are usually unstable and coarsen in time, except when wetting interactions are able to damp long-wave perturbations, which makes the instability Turing-like. In most of these cases, the zero wave number remains marginally stable [16,18], which should lead to the formation of unexpected patterns due to nonlinear couplings between critical and zero modes [19].

In contrast, one may think that the instability of the deposited layer and the formation of self-organized nanophases occurs in far from thermal equilibrium conditions and results from dynamics combining nongradient terms and gradient ones, similarly to the case of microstructures developing in surface chemical reactions [20]. Furthermore, it should occur in both monatomic layers and binary epilayers, as suggested in $[21,22]$ and $[23,24]$, respectively. It is why I proposed a reaction-diffusion model to describe, at the nanoscale, structure formation and texture evolution in an adsorbed monolayer on a substrate $[25,26]$. With this model, it has been shown that, even in monocomponent films, the competition between atomic deposition and the underlying instability of an adsorbed atomic layer may stabilize nanoscale spatial patterns, already in the first deposited layers. These patterns correspond to regular spatial distributions of high- and low-coverage domains, which may induce corresponding distributions of grains with different orientations or symmetries, and serve as templates for the later stages of film texture evolution. Patterns with different symmetries may be selected according to the relative values of experimental parameters such as deposition rate, substrate temperature, and atomic mobility. In systems with isotropic diffusion, successive transitions between hexagonal arrays of dots and striped nanostructures are predicted for increasing coverage or concentration. In this approach, pattern formation has a dynamical origin, in contrast to methods based on gradient dynamics, which ultimately result in the minimization of a potential [9-12,18].

This model has been extended to include growth effects in the third dimension to describe how the nanostructures may extend into the bulk of the growing film [27]. In this case the instability mechanism is only one among various instability mechanisms one may think of [28]. Another extension has been made on incorporating long-range interactions between deposited atom clusters mediated by the substrate [29]. In this case cluster-cluster interactions slightly favor stripes. The length scale of natural self-organized structures has been found to be in the range of tens of nanometers. Imposition of a substrate periodic strain field by subsurface interfacial dislocations has also been considered and has been shown to dramatically change the self-organized pattern and its length scale. Qualitative agreement between these predictions [29] and experimental observations on self-organized Ge quantum dots on Si substrate has been obtained [30,31]. This model has also been rederived, including more general desorption processes, and has been shown to generate highly nonlinear structures, corresponding to regular arrays of low-coverage holes in high-coverage background or to regular arrays of dots in a low-coverage background, as well as stable isolated localized structures [32].

Up to now the simplest deposition processes have been considered. They depend linearly on the atomic coverage of the growing film and may correspond to sputtering or laserassisted deposition [25,26]. This linear dependence has two effects. On the one side its lowers the instability temperature and, on the other side, it restricts the wavelength range for unstable spatial modes to a finite domain around a critical one. In this case, the nonlinear part of the dynamics, which defines pattern selection and stability through selective mode couplings, comes from the atomic diffusion in the first deposited layers only. However, in chemical vapor deposition, adsorption-desorption mechanisms are more complex [33] and the process is clearly a nonequilibrium one [34]. In fact, it is the competition between nonlinearities arising, on the one side, from the chemical reaction kinetics and, on the other side, from surface diffusion, which should determine pattern formation, selection, and stability in the deposited layer. It is why the aim of this paper is to extend the previous model to the case of chemical vapor deposition and to analyze how nonlinearities resulting from this process may affect the properties of the nanostructures described earlier $[25,26]$.

The paper is organized as follows. First, the dynamics of a deposited atomic layer on a substrate is reviewed and a reaction-diffusion model appropriate for chemical vapor deposition processes is derived in Sec. II. The stability of uniform deposited layers is analyzed in Sec. III. The weakly nonlinear dynamics is analyzed close to instability and the resulting pattern formation and selection beyond instability are analyzed for different types of nonlinearities and diffusion coefficients in Sec. IV. The possibility of experimental realization of the proposed instability mechanism is discussed in Sec. V. Finally, a summary and perspectives for future work are presented in Sec. VI.

\section{DYNAMICS OF A DEPOSITED LAYER ON A SUBSTRATE}

As already discussed in previous work, the evolution of a monatomic layer, deposited on a substrate, may be described by a continuous dynamical model of the reaction-diffusion type [25,26]. Relevant examples of such systems are Al or $\mathrm{Cu}$ layers deposited on $\mathrm{Si}$ substrates or $\mathrm{SiO}_{2}$ and $\mathrm{TiN}$ layers deposited on $\mathrm{Ti}$ or $\mathrm{Al}$ substrates. In such cases, the dynamics is governed by atomic adsorption, desorption, and reaction on the substrate, as well as by diffusion or transport. For a sufficiently small lattice misfit between film and substrate, elasticity and stress effects may be neglected and the film evolution may be described by atomic coverage dynamics only. Among the examples listed above, this may be the case for $\mathrm{Al}$ on $\mathrm{Si}(111)$ [35], $\mathrm{Cu}$ on $\mathrm{Si}(100)$ [36], or $\mathrm{Cu}$ on $\mathrm{Si}$, where the $\mathrm{Cu}(111)$ fcc plane is identical to the $\mathrm{Si}(0001)$ hcp plane and a perfect match can be achieved. Misfit strains may also be strongly reduced through domain matching, as in $\mathrm{Cu}$ on $\mathrm{TiN} \mid \mathrm{Si}$ and $\mathrm{TiN}$ on $\mathrm{Si}(100)$ [37], or buffer layers [38]. When misfit strains cannot be neglected, they may be relieved by surface deformation or dislocation nucleation and 
the deposited layer dynamics has to be coupled with the film elasticity. How this may affect nanostructure formation in the spirit of our adsorption-desorption-diffusion model is discussed elsewhere $[11,12,39,40]$. Let me recall that, for this model, the corresponding kinetic equation has been derived in the framework of chemical kinetics and has the following structure [26]:

$$
\partial_{t} c=R(c)-\nabla \cdot \mathbf{J},
$$

where $c=c(\mathbf{r}, t)$ is the local atomic coverage of the substrate, which is defined as the average occupancy number, or average atom number per lattice site; $R(c)$ represents reaction terms; and $\mathbf{J}$ is the atomic mass current in the deposited layer.

\section{A. Dynamics of chemical vapor deposition}

In chemical vapor deposition (CVD), typical procedures consist in the deposition of precursor molecules, which contain the active species of the growing film, on the substrate. These molecules dissociate through chemical reactions and liberate the active species atoms that remain adsorbed on the substrate to form the film. Some of them may recombine and desorb. Precursor dissociation may be autocatalytic and the chemical scheme of the process is of the type

$$
\begin{aligned}
P_{g}+n *(+m C) & \rightarrow P(+m C), \quad P+p C \rightarrow q C, \\
r C & \rightarrow B, \quad B \rightarrow B_{g}+r *,
\end{aligned}
$$

where the asterisk represents vacant lattice sites, the subscript $g$ represents gas phase concentration, $P$ represents precursor molecules, and $C$ is the active species that forms the film and also recombines in desorbing molecules $B$. For large Knudsen number, the precursor transport is ballistic and one may neglect flow transport processes of the precursor, its interaction with the surface being the dominant process. Then $C$ is the only diffusing species and the kinetic rate equations for the corresponding coverage may be written as

$$
\begin{aligned}
& \partial_{t} P=\alpha(1-C)^{n} C^{m}-\rho P C^{p}, \\
& \partial_{t} C=\rho P C^{p}-\beta C^{r}-\nabla \cdot \mathbf{J}, \quad \partial_{t} B=\beta C^{r}-\kappa B .
\end{aligned}
$$

In this dynamical system, the reaction rates are directly inferred from the reaction scheme (2) and are proportional to the concentrations (expressed here in lattice coverage) of each active species up to a power corresponding to its reaction order [41]. Here $\alpha, \beta, \rho$, and $\kappa$ are kinetic constants. They are functions of the physical parameters of the reaction mechanisms and have to be evaluated from experiment or atomistic simulations. In addition, $n$ represents the number of lattice sites required for the adsorption of a $P$ molecule, $m$ represents the autocatalytic nature of this process, and $r$ is the order of the recombination reaction with respect to $C$ in the desorption process. Nontrivial uniform steady states are given by $B_{0}=\frac{\beta}{\kappa} C_{0}^{r}, P_{0}=\frac{\beta}{\rho} C_{0}^{r-p}$, and $\left(1-C_{0}\right)^{n} C_{0}^{m-r}=\frac{\beta}{\alpha}$ (with $n \geqslant 1$ and $r \geqslant 2$ in nontrivial cases).

Many metal CVD deposition are autocatalytic, such as, for example, $\mathrm{Cu}$ deposition on $\mathrm{SiO}_{2}$ [42]. Autocatalysis is also associated with the fact that deposition is enhanced by already deposited material, as in $\mathrm{Si}, \mathrm{Cu}$, TiN, or Pt deposition [43]. The surface reaction controls the deposition rate at low temperatures and pressure when the precursor diffuses rapidly to the surface. In contrast, mass transport controls the rate at high temperature and high pressure when the precursor diffuses slowly to the surface. In situations where the surface reaction kinetics is the rate limiting process, the precursor $P$ and desorbed $B$ species may be adiabatically eliminated in (3), which leads to the following kinetic equation for the active species coverage:

$$
\partial_{t} c=\alpha(1-c)^{n} c^{m}-\beta c^{r}-\nabla \cdot \mathbf{J}(c) .
$$

When $m<r$, the order of autocatalysis is higher for adsorption than for desorption, which makes the latter less efficient since coverage varies between 0 and 1 [26]. A spatially uniform finite-coverage steady state should thus be expected. Effectively, in this case, the steady state is given by

$$
\left[\alpha(1-c)^{n}-\beta c^{r-m}\right] c^{m}=0
$$

and there is one trivial $c_{0}=0$ and one nontrivial $c_{0} \neq 0$ solution. With the evolution of homogeneous perturbations $\sigma$ of the trivial solution being given by

$$
\partial_{t} \sigma=\left[\alpha(1-\sigma)^{n}-\beta \sigma^{r-m}\right] \sigma^{m} \simeq \alpha \sigma^{m},
$$

it turns out that this solution is unstable. In contrast, with the linear evolution of homogeneous perturbations of the nontrivial solution being

$$
\partial_{t} \sigma=-n\left[\alpha\left(1-c_{0}\right)^{n-1}+(r-m) \beta c_{0}^{r-m-1}\right] c_{0}^{m} \sigma<0,
$$

this steady state is stable.

When $m=r$, besides a trivial steady state, there is one nontrivial steady state if $\beta<\alpha$. In this case, the trivial steady state is unstable while the nontrivial one is stable.

When $m>r$, desorption is favored over adsorption and a state with zero coverage should remain as such. This does not rule out that a sufficiently large perturbation could lead to film growth. Effectively, besides the trivial steady state, for

$$
\frac{\beta}{\alpha}<\frac{n^{n}(m-r)^{m-r}}{(m+n-r)^{m+n-r}}=\left.\left(\frac{\beta}{\alpha}\right)\right|_{\max },
$$

there a two nontrivial ones $c_{ \pm}$given by

$$
\alpha\left(1-c_{ \pm}\right)^{n} c_{ \pm}^{m-r}-\beta=0 .
$$

The stability of the trivial solution is given by

$\partial_{t} \sigma=\left[\alpha(1-\sigma)^{n} \sigma^{m-r}-\beta\right] \sigma^{r}=-\beta\left[1-O\left(\sigma^{n \geqslant 1}\right)\right] \sigma^{r}$,

which shows that it is linearly or marginally stable. If one defines $c^{*}=\frac{m-r}{m+n-r}$ as the steady state corresponding to $\left.\left(\frac{\beta}{\alpha}\right)\right|_{\max }\left[\right.$ for $\left.\left.\left(\frac{\beta}{\alpha}\right)\right|_{\max }, c_{+}=c_{-}=c^{*}\right]$, the linear stability of the nontrivial steady states is given by

$$
\beta(n+m-r) \frac{c^{*}-c_{ \pm}}{c_{ \pm}\left(1-c_{ \pm}\right)}<0
$$

and the state $c_{-}<c^{*}$ is unstable while the state $c_{+}>c^{*}$ is stable. These behaviors are illustrated in Fig. 1, where steady states for the different regimes are displayed.

\section{B. Transport}

It is now necessary to model atomic motion adequately. As already mentioned elsewhere [26,32], the conditions required to apply Fick's law are not met in adsorbed layers, where interactions between adsorbed particles may be important. 


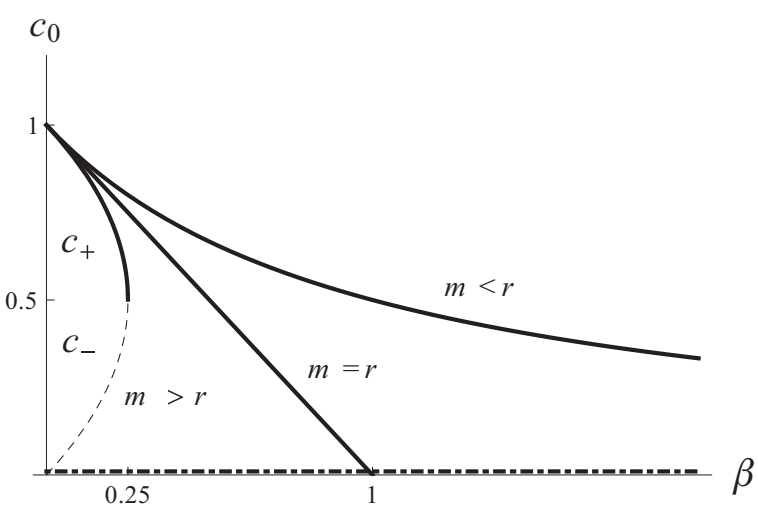

FIG. 1. Examples of uniform steady states of Eq. (4) for $m>r$ ( $m=r+1), m=r$, and $m<r(m=r-1)$ versus $\beta$ with $\alpha=1$ (solid lines correspond to stable states versus uniform perturbations, dashed lines correspond to unstable states, and the mixed line is for the $c=0$ state, the stability of which is different in each regime).

In this case, diffusion is governed by a spatially varying chemical potential and linear nonequilibrium thermodynamics provides the necessary tools to describe this situation [44]. The Gibbs-Duhem and Onsager reciprocity relations hold, and, as a result [45], for isothermal diffusion, which is the case here, the atomic mass current of the active species is proportional to the gradient of the chemical potential. Since the chemical potential is the functional derivative of the free energy, one has

$$
\mathbf{J}=-L \nabla \frac{\delta \mathcal{F}}{\delta c},
$$

where $\mathcal{F}$ is the free energy of the adsorbed layer formed by the active species $C$ and $L$ is the surface mobility. In the mean field approximation, an explicit expression for the free energy may be obtained, which reads $[26,32]$

$$
\mathcal{F}=\int_{S} d \mathbf{r}\left[k_{B} T f(\mathbf{r})-\frac{1}{2} \epsilon_{0} c(\mathbf{r})^{2}+\frac{1}{2} \xi_{0}^{2}|\nabla c(\mathbf{r})|^{2}\right],
$$

where $c(\mathbf{r})$ is the local coverage and $f(\mathbf{r})=[1-c(\mathbf{r})] \ln [1-$ $c(\mathbf{r})]+c(\mathbf{r}) \ln c(\mathbf{r})-\epsilon_{0} c$. For nearest-neighbor attractive interactions between deposited atom, $\epsilon_{0}=\gamma \epsilon$ and $\xi_{0}^{2}=\gamma \epsilon l^{2}$, where $\gamma$ is the lattice coordination number, $\epsilon$ is the pair interaction energy, and $l$ is the lattice constant. Interaction between the substrate and adsorbed particles may be introduced through an extra contribution equal to $\epsilon_{s} c(\mathbf{r})$ in the local free energy. The local chemical potential may then be easily obtained from the resulting free energy and is written

$$
\mu(\mathbf{r})=\frac{\delta \mathcal{F}}{\delta c}=\epsilon_{s}-\epsilon_{0} c(\mathbf{r})+k_{B} T \ln \frac{c(\mathbf{r})}{1-c(\mathbf{r})}+\xi_{0}^{2} \nabla^{2} c(\mathbf{r}) .
$$

This is the equation of state of the system, which defines the coverage as a function of temperature, interaction energies, etc. Let us recall that thermodynamic stability of a state $c(\mathbf{r})$ requires

$$
\frac{\partial^{2} f}{\partial c^{2}}=-\epsilon_{0}+k_{B} T \frac{1}{c(1-c)}>0
$$

and this condition is always satisfied for $\epsilon_{0}<4 k_{B} T$, or $T>T_{c}$, with $\epsilon_{0}=4 k_{B} T_{c}$.
However, for $T<T_{c}$, states in the range

$$
\frac{1}{2}\left[1-\sqrt{1-\frac{T}{T_{c}}}\right]<c<\frac{1}{2}\left[1+\sqrt{1-\frac{T}{T_{c}}}\right]
$$

are thermodynamically unstable. Hence, in this range and without reacting terms, single homogeneous phases are unstable and, due to coarsening, the system separates into two distinct phases, one with low coverage $\left(c<\frac{1}{2}\right)$ and one with high coverage ( $c>\frac{1}{2}$ ). Here $T=T_{c}$ defines the critical point below which phase separation occurs in the adsorbed layer. The corresponding critical coverage and chemical potential are $c_{c}=\frac{1}{2}$ and $\mu_{c}=\epsilon_{s}-2 k_{B} T_{c}$, respectively.

Furthermore, the Onsager coefficient $L$ may be written as $L=\frac{D_{\text {diff }}}{k_{B} T}$, where $D_{\text {diff }}$ is the surface diffusion coefficient. Its coverage and temperature dependence are crucial issues for the development of spatial instabilities. In a first approximation, it is usually considered as a constant, which is appropriate for small deviations from steady uniform coverages. However, for high-coverage variations and, according to the dynamical processes involved in atomic displacements and to the presence of clusters or other sinks, its coverage dependence should be taken into account. For example, expressions such as $D_{\text {diff }}=$ $D \times c(1-c)$ may be more appropriate for hopping types of motion, with $D \propto \exp -(\Omega / T)$, where $\Omega$ is related to the activation energy of atomic jumps.

\section{STABILITY OF UNIFORM DEPOSITED LAYERS}

Since the presence of reaction terms is expected to modify the stability of uniform deposited layers, it will be studied on combining the kinetic equation (4) with (13). The dynamics of the active species coverage then becomes

$$
\begin{aligned}
\partial_{t} c(\mathbf{r}, t)= & R[c(\mathbf{r}, t)]-\nabla\left[\frac { D _ { \mathrm { diff } } } { k _ { B } T } \cdot \nabla \left(\epsilon_{0} c(\mathbf{r}, t)\right.\right. \\
& \left.\left.-k_{B} T \ln \frac{c(\mathbf{r}, t)}{1-c(\mathbf{r}, t)}+\xi_{0}^{2} \nabla^{2} c(\mathbf{r}, t)\right)\right],
\end{aligned}
$$

where $R[c(\mathbf{r}, t)]=\alpha[1-c(\mathbf{r}, t)]^{n} c(\mathbf{r}, t)^{m}-\beta c(\mathbf{r}, t)^{r}$.

Note that the case $n=1, m=0$, which corresponds to direct absorption, without precursor molecule dissociation, and in the absence of chemical reaction with the substrate, has already been extensively studied in previous work [26,32]. I will thus concentrate on more general cases, which are relevant for CVD.

\section{A. The case $m<r$}

Let us consider first the case $m<r$, where the reaction part of the dynamics admits a single stable uniform steady state $c_{0}$. The linear stability analysis of this steady state versus small nonuniform perturbations $\sigma(\mathbf{r}, t)=c(\mathbf{r}, t)-c_{0}$ is studied through its linear evolution equation, with $D_{\text {diff }}=$ $D \times c(1-c)$ :

$$
\begin{aligned}
\partial_{t} \sigma(\mathbf{r}, t)= & -\Gamma \sigma(\mathbf{r}, t)-\frac{D}{k_{B} T} \nabla^{2}\left[\epsilon_{0} c_{0}\left(1-c_{0}\right)-k_{B} T\right. \\
& \left.+\xi_{0}^{2} c_{0}\left(1-c_{0}\right) \nabla^{2}\right] \sigma(\mathbf{r}, t)
\end{aligned}
$$


or in a Fourier transform

$$
\partial_{t} \sigma(\mathbf{q}, t)=-\left[\Gamma+\hat{D}_{0}\left(1-\frac{T}{\hat{T}_{0}}-l^{2} q^{2}\right) q^{2}\right] \sigma(\mathbf{q}, t),
$$

where $\Gamma=-\left.\frac{d R(c)}{d c}\right|_{c_{0}}, \quad \hat{D}_{0}=\frac{D \hat{T}_{0}}{T}, \hat{T}_{0}=4 T_{c} c_{0}\left(1-c_{0}\right)$, and $l^{2}=\frac{\xi_{0}^{2}}{\epsilon_{0}}$.

Instability may occur if $T<\hat{T}_{0}$ and its threshold is given by $q_{i}^{2}=\frac{1}{2 l^{2}}\left(1-\frac{T_{0}^{i}}{\hat{T}_{0}}\right)$ and $\Gamma=\frac{\hat{D}_{0} \hat{T}_{0}}{4 l^{2} T_{0}^{i}}\left(1-\frac{T_{0}^{i}}{\hat{T}_{0}}\right)^{2}$. Uniform steady states are thus unstable for

$T<4 T_{c} c_{0}\left(1-c_{0}\right)\left[1-\frac{\Gamma l^{2}}{D}\left(\sqrt{1+\frac{2 D}{\Gamma l^{2}}}-1\right)\right]=T_{0}^{i}$

where $T_{0}^{i}$ is the instability threshold temperature. Note that the deposition process lowers the threshold temperature with respect to the pure spinodal case. The system will thus become unstable on lowering $T$ below $T_{0}^{i}$.

\section{B. The case $m>r$}

When $m>r$, the reaction part of the dynamics admits one trivial and two nontrivial uniform steady states. One thus has to test now the stability versus inhomogeneous perturbations of the states that are stable versus homogeneous ones. For the sake of concreteness, let me consider as an example the case $n=1$, $m=2, r=1$, which corresponds to $R(c)=\alpha(1-c) c^{2}-\beta c$. If $\frac{\beta}{\alpha}>\frac{1}{4}$, the only possible steady state is zero and no steady deposited layer forms since evaporation dominates deposition. In contrast, if $\frac{\beta}{\alpha}<\frac{1}{4}$ there are three possible uniform steady states, $c_{0}=0$ and $c_{ \pm}=\frac{1}{2}\left(1 \pm \sqrt{1-4 \frac{\beta}{\alpha}}\right)$.

The linear evolution of perturbations of the trivial steady state $c_{0}=0$ is given by

$$
\partial_{t} c(\mathbf{r}, t)=-\beta c(\mathbf{r}, t)+D \nabla^{2} c(\mathbf{r}, t),
$$

showing that this state is linearly stable. The linear evolution of perturbations of the nontrivial steady states $c_{ \pm}$is given by

$$
\begin{aligned}
\partial_{t} \sigma(\mathbf{q}, t)= & \alpha c_{ \pm}\left(1-2 c_{ \pm}\right) \sigma(\mathbf{q}, t) \\
& +\hat{D}_{ \pm} q^{2}\left[1-\frac{T}{\hat{T}_{ \pm}}-l^{2} q^{2}\right] \sigma(\mathbf{q}, t),
\end{aligned}
$$

where $\hat{D}_{ \pm}=\frac{D \hat{T}_{ \pm}}{T}$ and $\hat{T}_{ \pm}=4 T_{c} c_{ \pm}\left(1-c_{ \pm}\right)$. As already known, $c_{-}$is unstable since $1-2 c_{-}>0$. In contrast, $c_{+}$ is stable provided $T>\hat{T}_{+}$. When $T<\hat{T}_{+}$, it may become unstable versus nonuniform perturbations. For $c_{+}=\frac{1}{2}$, it is unstable for all perturbations with wave numbers in the domain defined by $0<q^{2}<\left(1-\frac{T}{\hat{T}_{+}}\right) \frac{1}{l^{2}}$, which extends to 0 as in spinodal decomposition. For $c_{+}>\frac{1}{2}$, this instability domain does not extend to zero. Since the maximum growth rate corresponds to $q_{m}^{2}=\left(1-\frac{T}{\hat{T}_{+}}\right) \frac{1}{2 l^{2}}$, the instability threshold is defined by $\alpha c_{+}\left(2 c_{+}-1\right)=\frac{D}{4 l^{2}} \frac{\left(\hat{T}_{+}-T_{+}^{i}\right)^{2}}{\hat{T}_{+} T_{+}^{i}}$ and $c_{+}$is unstable for

$$
T<4 T_{c} c_{+}\left(1-c_{+}\right)\left[1-\Lambda\left(\sqrt{1+\frac{2}{\Lambda}}-1\right)\right]=T_{+}^{i}
$$

where $\Lambda=\frac{l^{2} \alpha c_{+}\left(2 c_{+}-1\right)}{D}$ and $T_{+}^{i}$ is the instability threshold temperature for the actual reaction scheme. Furthermore, the band of unstable wave numbers is defined by

$$
\begin{aligned}
& q_{m}^{2}\left[1-\sqrt{1-\frac{T}{T_{+}^{i}}\left(\frac{\hat{T}_{+}-T_{+}^{i}}{\hat{T}_{+}-T}\right)^{2}}\right] \\
& \quad<q^{2}<q_{m}^{2}\left[1+\sqrt{1-\frac{T}{T_{+}^{i}}\left(\frac{\hat{T}_{+}-T_{+}^{i}}{\hat{T}_{+}-T}\right)^{2}}\right] .
\end{aligned}
$$

\section{WEAKLY NONLINEAR DYNAMICS AND PATTERN SELECTION BEYOND INSTABILITY}

Having determined the instability conditions for uniform deposited layers versus short-wave spatial modes, nonlinear analysis is needed to predict which kind of patterns could saturate the instability. The nonlinear evolution equation for perturbations $\sigma(\mathbf{r}, t)$ of a uniform steady state $c_{0}$, derived from Eq. (16), may be written as

$$
\begin{aligned}
\partial_{t} \sigma(\mathbf{r}, t)= & -\mathcal{R}\left[c_{0}, \sigma(\mathbf{r}, t)\right]-\nabla\left[\frac{D\left[c_{0}, \sigma(\mathbf{r}, t)\right]}{k_{B} T}\right. \\
& \left.\times\left(\epsilon_{0}-\frac{k_{B} T}{c(\mathbf{r}, t)[1-c(\mathbf{r}, t)]}+\xi_{0}^{2} \nabla^{2}\right)\right] \cdot \nabla \sigma(\mathbf{r}, t) .
\end{aligned}
$$

Close to the instability point, a separation of time and space scales occurs, which is due to the fact that the real parts of the eigenvalues of the unstable modes tend to zero or become positive, whereas in the case of stable modes they remain finite. Hence the evolution of the unstable modes is much slower than the evolution of the stable ones. This is equivalent to the critical slowing down phenomenon in equilibrium phase transitions. As a result, the faster evolution of the stable modes usually allows one to perform their adiabatic elimination from the dynamics. As a result, close to instability points, the dynamics is governed by the evolution of the unstable modes only. This reduction leads to a dramatic simplification of the dynamics that nevertheless captures the asymptotic properties of the systems evolution. This procedure is now well documented and has been applied in a large variety of complex nonlinear systems [1,46-48].

Close to instability, the dynamics may then be expanded in a series expansion in powers of $\sigma(\mathbf{r}, t)$, limited to the first nonlinear stabilizing term, which usually corresponds to cubic nonlinearities. This weakly nonlinear dynamics usually allows an accurate description of pattern formation, selection, and stability up to a finite distance beyond instability [49]. Such weakly nonlinear analysis will now be performed for explicit expressions of the diffusion coefficient and different types of reaction processes.

\section{A. Weakly nonlinear analysis for $D=D_{0} c(1-c)$ and $m<r$}

In this case, the weakly nonlinear evolution equation, derived from Eq. (23), and limited to cubic nonlinearities is written

$$
\partial_{t} \sigma(\mathbf{r}, t)=-\left[\Gamma+\hat{D}_{0}\left(1-\frac{T}{\hat{T}_{0}}+l^{2} \nabla^{2}\right) \nabla^{2}\right] \sigma(\mathbf{r}, t)
$$




$$
\begin{aligned}
& -\Gamma_{2} \sigma^{2}(\mathbf{r}, t)-\frac{\hat{D}_{0}\left(1-2 c_{0}\right)}{c_{0}\left(1-c_{0}\right)} \\
& \times \nabla\left\{\sigma(\mathbf{r}, t) \cdot \nabla\left[\sigma(\mathbf{r}, t)+l^{2} \nabla^{2} \sigma(\mathbf{r}, t)\right]\right\} \\
& -\Gamma_{3} \sigma^{3}(\mathbf{r}, t)+\frac{\hat{D}_{0}}{c_{0}\left(1-c_{0}\right)} \\
& \times \nabla\left\{\sigma^{2}(\mathbf{r}, t) \cdot \nabla\left[\sigma(\mathbf{r}, t)+l^{2} \nabla^{2} \sigma(\mathbf{r}, t)\right]\right\},
\end{aligned}
$$

where $\quad \Gamma=-\left.\frac{d R(c)}{d c}\right|_{c_{0}}, \quad \Gamma_{2}=-\left.\frac{1}{2} \frac{d^{2} R(c)}{d c^{2}}\right|_{c_{0}}, \quad$ and $\quad \Gamma_{3}=$ $-\left.\frac{1}{6} \frac{d^{3} R(c)}{d c^{3}}\right|_{c_{0}}$.

As discussed in the preceding section, uniform coverage is unstable for $T<T_{0}^{i}$ versus nonuniform perturbations. Fourier modes with $q=q_{i}$ have the maximum growth rate and may be expected to dominate the system evolution and generate spatial patterns. Close to instability, pattern evolution and selection may be studied through their amplitude equations [49,50]. In this analysis, the simplest autocatalytic processes will be considered (where $n=1, m=1, r=2, c_{0}=\frac{\alpha}{\alpha+\beta}, \Gamma=\alpha$, $\Gamma_{2}=\alpha+\beta, \Gamma_{3}=0$, and $n=1, m=1, r=1$, with $c_{0}=$ $\frac{\alpha-\beta}{\alpha}, \Gamma=\alpha-\beta, \Gamma_{2}=\alpha, \Gamma_{3}=0$ ) to allow an explicit analysis of the balance between reaction and diffusion generated nonlinearities. The different types of patterns that may arise from this dynamics, as well as their stability, will now be discussed.

\section{Stripes}

The simplest patterns, able to develop on an isotropic substrate, correspond to stripes such that $\sigma(\mathbf{r}, t)=A_{1}(\mathbf{r}, t) e^{i q_{i} x}+$ $A_{1}^{*}(\mathbf{r}, t) e^{-i q_{i} x}$. On inserting this expression in (24) and neglecting contributions coming from higher harmonics [e.g., $\left.\left.\exp \left( \pm i n q_{i} x\right)\right|_{n \geqslant 1}\right]$, their amplitude equation derived from Eq. (24) is written

$$
\begin{aligned}
\tau \partial_{t} A_{1}(\mathbf{r}, t)= & \epsilon A_{1}(\mathbf{r}, t)+\lambda\left(\nabla_{x}+\frac{i}{2 q_{i}} \nabla_{y}^{2}\right)^{2} A_{1}(\mathbf{r}, t) \\
& -\kappa\left|A_{1}(\mathbf{r}, t)\right|^{2} A_{1}(\mathbf{r}, t)
\end{aligned}
$$

where $\epsilon=\frac{T_{0}^{i}-T}{T}, \quad \tau=\frac{4 l^{2}}{D} \frac{T_{0}^{i} \hat{T}_{0}}{\hat{T}_{0}^{2}-\left(T_{0}^{i}\right)^{2}}, \quad \lambda=\frac{8 l^{2} \hat{T}_{0}}{\hat{T}_{0}-T_{0}^{i}}, \quad$ and $\quad \kappa=$ $\frac{T_{0}^{i}}{T c_{0}\left(1-c_{0}\right)}$.

In the vicinity of instability $\epsilon$ is small and all terms of this equation scale as $\epsilon^{3 / 2}\left(A\right.$ scales as $\epsilon^{1 / 2}$, time as $\epsilon^{-1}, x$ as $\epsilon^{-1 / 2}$, and $y$ as $\epsilon^{-1 / 4}$ ) [46]. In this sense, this equation may also be obtained from a multiple scale analysis. This method is standard now and technical details may be found in various references [49,50].

Note that this procedure is only justified for a finite range of unstable wave numbers close to the critical one. Too far in the unstable domain, the unstable range may become sufficiently large to include harmonics of the critical mode and even the zero-wave-number mode. In this case, the weakly nonlinear analysis breaks down. An exception occurs when the set of unstable modes contains the zero mode and a separate finite band of modes around a nonzero critical one. Amplitude equations may then be derived for critical and zero modes that are nonlinearly coupled and this coupling may strongly affect pattern selection and stability [19].

In the present case, steady state stripe patterns of amplitude $\left|A_{1}\right|^{2}=\left|A_{s}\right|^{2}=\frac{\epsilon}{\kappa}=\frac{T_{0}^{i}-T}{T_{0}^{i}} c_{0}\left(1-c_{0}\right)$ should develop for
$T \leqslant T_{0}^{i}$. However, stripe patterns are unstable versus hexagonal ones in a finite-temperature domain as discussed in [49] and in the next section.

\section{Hexagons}

Hexagonal planforms, such that $\sigma(\mathbf{r}, t)=$ $\sum_{i=1}^{3} A_{i}(\mathbf{r}, t) e^{i \mathbf{q}_{i} \cdot \mathbf{r}}+$ c.c. with $\sum_{i=1}^{3} \mathbf{q}_{i}=0$ and $\left|\mathbf{q}_{1}\right|=\left|\mathbf{q}_{2}\right|$ $=\left|\mathbf{q}_{3}\right|=q_{i}$, are described by the following amplitude equation:

$$
\begin{aligned}
\tau \partial_{t} A_{i}= & \epsilon A_{i}+\frac{\lambda}{q_{i}^{2}}\left(\mathbf{q}_{i} \cdot \nabla\right)^{2} A_{i}+2 \nu A_{i+1}^{*} A_{1+2}^{*} \\
& -\kappa A_{i}\left(\left|A_{i}\right|^{2}+\rho\left|A_{i+1}\right|^{2}+\rho\left|A_{i+2}\right|^{2}\right),
\end{aligned}
$$

where $v=-\tau(\alpha+\beta)+\frac{T_{0}^{i}\left(1-2 c_{0}\right)}{T c_{0}\left(1-c_{0}\right)}$ for $r=2, \quad v=-\tau \alpha+$ $\frac{T_{0}^{i}\left(1-2 c_{0}\right)}{T c_{0}\left(1-c_{0}\right)}$ for $r=1$, and $\rho=2$. Strictly speaking, $v$ should scale as $\sqrt{\epsilon}$ for consistency. This is not always the case, but this equation is nevertheless used when $v$ may be considered small $[47,51,52]$. Common analysis shows that these equations admit steady state solutions corresponding to hexagonal planforms of amplitude

$$
\left|A_{1}\right|=\left|A_{2}\right|=\left|A_{3}\right|=R_{ \pm}=\frac{1}{5 \kappa}\left(|\nu| \pm \sqrt{\nu^{2}+5 \kappa \epsilon}\right) .
$$

These solutions may appear subcritically for $\epsilon>-\frac{v^{2}}{5 \kappa}$. Here $R_{-}$is unstable and $R_{+}$is stable provided $\epsilon<\frac{16 v^{2}}{\kappa}$ [49]. Its existence and stability range are thus given by

$T_{0}(h-)=T_{0}^{i}(1-16 \Delta)<T<T_{0}^{i}\left(1+\frac{\Delta}{5}\right)=T_{0}(h+)$,

where $\Delta=\frac{v^{2}}{\kappa}$. The sign of $v$ is important since it determines the type of hexagons that are stable in this domain. Effectively, for $v>0$ they correspond to $H_{+}$hexagons (with the amplitude maxima at the hexagon centers), while for $v<0$, they correspond to $H_{-}$hexagons (with the amplitude minima at the hexagon centers) [49]. Since in this example $1-2 c_{0}=\frac{\beta-\alpha}{\alpha+\beta}$, $v$ is negative for $\beta<\alpha$, i.e., for high coverage $\left(c_{0}>\frac{1}{2}\right)$. The selected pattern should thus correspond to $H_{-}$hexagons with minimum coverage at the center of the hexagons (like a triangular lattice of holes in the film). If $c_{0}<\frac{1}{2}$ or $\beta>\alpha$, i.e., for low coverage, $v$ is positive only if $\frac{\alpha}{\beta}<1-\frac{T}{T_{0}^{i}} \frac{\hat{T}_{0}-T_{0}^{i}}{\hat{T}_{0}+T_{0}^{i}}$ for $r=2$ or $\frac{\alpha}{\beta}<2-\frac{T}{T_{0}^{i}} \frac{\hat{T}_{0}-T_{0}^{i}}{\hat{T}_{0}+T_{0}^{i}}$ for $r=1$. In this case the selected pattern should be like a triangular lattice of dots on a low-coverage background. This result is of course the same as the one discussed in [26]. The only difference is that the limit between 0 and $\pi$ hexagons is shifted by reactive terms of the dynamics that favor the formation of $H_{-}$hexagons.

As mentioned in the preceding section, striped patterns may be unstable versus hexagonal planforms. Hexagonal perturbations of stripes of amplitude $\left|A_{s}\right|=\frac{\epsilon}{\kappa}$ and wave vector $\mathbf{q}_{1}$ are such that $\sigma(\mathbf{r}, t)=\left(A_{s}+a_{1}\right) e^{i \mathbf{q}_{1} \cdot \mathbf{r}}+a_{2} e^{i \mathbf{q}_{2} \cdot \mathbf{r}}+a_{3} e^{i \mathbf{q}_{3} \cdot \mathbf{r}}+$ c.c. Their linear evolution equations are given by

$$
\begin{aligned}
& \tau \partial_{t} a_{1}=\epsilon a_{1}-3 \kappa\left|A_{s}\right|^{2} a_{1}=-2 \epsilon a_{1}, \\
& \tau \partial_{t} a_{2}=\epsilon a_{2}+2 v a_{3}^{*} A_{s}^{*}-2 \kappa a_{2}\left|A_{s}\right|^{2}=-\epsilon a_{2}+2 v a_{3}^{*} A_{s}^{*}, \\
& \tau \partial_{t} a_{3}^{*}=\epsilon a_{3}^{*}+2 v a_{2} A_{s}-2 \kappa a_{3}^{*}\left|A_{s}\right|^{2}=-\epsilon a_{3}^{*}+2 \nu a_{2} A_{s}
\end{aligned}
$$


and the eigenvalues of the corresponding evolution matrix are given by $\omega=-2 \epsilon, \omega=-\epsilon-2 \nu \sqrt{\frac{\epsilon}{\kappa}}$, and $\omega=-\epsilon+2 \nu \sqrt{\frac{\epsilon}{\kappa}}$. Since the last root is negative only for $\epsilon>\frac{4 \nu^{2}}{\kappa}$, stripes are unstable for $0<\epsilon<\frac{4 v^{2}}{\kappa}$ and stable for $\epsilon>\frac{4 v^{2}}{\kappa}$. As a result, hexagons and stripes are simultaneously stable in the bistability domain defined by $\frac{4 v^{2}}{\kappa}<\epsilon<\frac{16 v^{2}}{\kappa}$ or $T_{h-}=$ $T_{0}^{i}(1-16 \Delta)<T<T_{0}^{i}(1-4 \Delta)=T_{s}$.

Note that, since $\rho=2$, square patterns are always unstable when described by such equations. As a result, pattern formation phenomena, discussed in this section, are qualitatively similar to the case described previously [26]: On decreasing temperature a sequence of transitions leads from uniform layers to hexagonal patterns and finally to stripes, with an intermediate bistability domain for hexagons and stripes. The effect of more complex reaction processes associated with chemical vapor deposition, which favor adsorption, is thus only quantitative and solely modifies the existence and stability ranges of the different patterns.

\section{B. Weakly nonlinear analysis for $D=D_{0} c(1-c)$ and $m>r$}

Let us consider now the case where adsorption processes are autocatalytic, with a higher degree of nonlinearity than adsorption ones. Corresponding uniform steady states and their linear stability have been described previously. As far as the nonlinear dynamics is concerned, two cases have to be considered.

\section{The case of critical coverage $c_{+}=\frac{1}{2}$}

This case will be illustrated for $n=1, m=2, r=1$, and $\alpha=4 \beta$, where $2 c_{+}-1=0$, and the nonlinear evolution of coverage perturbations is written

$$
\begin{aligned}
\partial_{t} \sigma(\mathbf{r}, t)= & -\frac{D T_{c}}{T}\left[1-\frac{T}{T_{c}}+l^{2} \nabla^{2}\right] \nabla^{2} \sigma(\mathbf{r}, t)-\frac{\alpha}{2} \sigma^{2}(\mathbf{r}, t) \\
& -\alpha \sigma^{3}(\mathbf{r}, t)+\frac{D T_{c}}{T}\left[1+l^{2} \nabla^{2}\right] \nabla\left[\sigma^{2}(\mathbf{r}, t) \cdot \nabla \sigma(\mathbf{r}, t)\right] .
\end{aligned}
$$

In a Fourier transform, for $T<T_{c}$, the band of unstable wave numbers $q$ is defined by $0<q^{2}<\frac{T_{c}-T}{T_{c} l^{2}}$, which includes zero as in spinodal decomposition. Hence the spatial gradient terms of the dynamics should induce coarsening of initially developing patterns since the dynamics would be of the genuine CahnHilliard type. However, the remaining nonlinear terms coming from the chemical part of the dynamics damp long-wavelength modes. They should thus not allow the growth of such modes nor coarsening. In contrast, they couple the most unstable short-wavelength modes and saturate their growth, providing a stabilizing mechanism for nanostructures with finite wave number. Among them, hexagons should be favored by the presence of quadratic nonlinearities. However, since the band of unstable wave vectors extends to zero, amplitude equations are not appropriate to discuss pattern and wavelength selection. In this case, they should be studied numerically.

\section{The case of high coverage $c_{+} \lesssim 1$}

This case corresponds to $m>r$ and $\left.\frac{\beta}{\alpha} \ll \frac{\beta}{\alpha}\right|_{\max }$. For example, for $n=1, m=2, r=1$, and $\alpha \gg 4 \beta, 2 c_{+}-1 \geqslant 0$ and the nonlinear evolution of coverage perturbations is written

$$
\begin{aligned}
\partial_{t} \sigma(\mathbf{r}, t)= & -\left[\alpha c_{+}\left(2 c_{+}-1\right)+\hat{D}_{+}\left(1-\frac{T}{\hat{T}_{+}}+l^{2} \nabla^{2}\right) \nabla^{2}\right] \\
& \times \sigma(\mathbf{r}, t)-\alpha\left(3 c_{+}-1\right) \sigma^{2}-\frac{\hat{D}_{+}\left(1-2 c_{+}\right)}{c_{+}\left(1-c_{+}\right)} \\
& \times\left[1+l^{2} \nabla^{2}\right] \nabla[\sigma(\mathbf{r}, t) \cdot \nabla \sigma(\mathbf{r}, t)]-\alpha \sigma^{3} \\
& +\frac{\hat{D}_{+}}{c_{+}\left(1-c_{+}\right)}\left[1+l^{2} \nabla^{2}\right] \nabla\left[\sigma^{2}(\mathbf{r}, t) \cdot \nabla \sigma(\mathbf{r}, t)\right] .
\end{aligned}
$$

Amplitude equations for different types of spatial patterns may then be derived from this equation in order to analyze their existence and stability, as in the discussion in Sec. IV A.

\section{Stripes}

Here also, stripes will be considered first as the simplest structure to arise from this dynamics. Their amplitude equations may be written, at lowest order in $\epsilon=\frac{T_{+}^{i}-T}{T}$, as

$$
\begin{aligned}
\tau_{+} \partial_{t} A_{1}(\mathbf{r}, t)= & \epsilon A_{1}(\mathbf{r}, t)+\lambda_{+}\left(\nabla_{x}+\frac{i}{2 q_{i}} \nabla_{y}^{2}\right)^{2} A_{1}(\mathbf{r}, t) \\
& -\left[3 \alpha_{+}+\kappa_{+}\right]\left|A_{1}(\mathbf{r}, t)\right|^{2} A_{1}(\mathbf{r}, t),
\end{aligned}
$$

where $\tau_{+}=\frac{4 l^{2}}{D} \frac{T_{+}^{i} \hat{T}_{+}}{\hat{T}_{+}^{2}-\left(T_{+}^{i}\right)^{2}}, \lambda_{+}=\frac{8 l^{2} \hat{T}_{+}}{\hat{T}_{+}-T_{+}^{i}}, \alpha_{+}=\frac{1}{c_{+}\left(2 c_{+}-1\right)} \frac{\hat{T}_{+}-T_{+}^{i}}{\hat{T}_{+}+T_{+}^{i}}$, and $\kappa_{+}=\frac{T_{+}^{i}}{T c_{+}\left(1-c_{+}\right)}$. Steady stripes of amplitude given by $\left|A_{1}\right|^{2}=A_{+}^{2}=\frac{\epsilon}{3 \alpha_{+}+\kappa_{+}}$may thus appear supercritically for $\epsilon>0$.

\section{Hexagons}

In contrast, amplitude equations for hexagonal or honeycomb planforms are written

$$
\begin{aligned}
\tau_{+} \partial_{t} A_{i}= & \epsilon A_{i}+\frac{\lambda_{+}}{q_{i}^{2}}\left(\mathbf{q}_{1} \cdot \nabla\right)^{2} A_{i}+2 \nu_{+} A_{i+1}^{*} A_{i+2}^{*} \\
& -\left(3 \alpha_{+}+\kappa_{+}\right) A_{i}\left(\left|A_{i}\right|^{2}+\rho\left|A_{i+1}\right|^{2}+\rho\left|A_{i+2}\right|^{2}\right) \\
& (i=1,2,3),
\end{aligned}
$$

where

$$
v_{+}=\frac{\left(1-3 c_{+}\right)}{c_{+}\left(2 c_{+}-1\right)} \frac{\hat{T}_{+}-T_{+}^{i}}{\hat{T}_{+}+T_{+}^{i}}+\frac{T_{+}^{i}\left(1-2 c_{+}\right)}{T c_{+}\left(1-c_{+}\right)}
$$

and $\rho=2$. It turns out that $v_{+}$is always negative, which leads to the formation of $\pi$ or negative hexagons only. Furthermore, using the results of standard pattern formation theory [49], these hexagons, of amplitude

$$
\left|A_{i}\right|=\frac{1}{5\left(3 \alpha_{+}+\kappa_{+}\right)}\left[\left|v_{+}\right|+\sqrt{\nu_{+}^{2}+5\left(3 \alpha_{+}+\kappa_{+}\right) \epsilon}\right]
$$

are stable in the range

$$
T_{+}(h-)=T_{+}^{i}\left(1-16 \Delta_{+}\right)<T<T_{+}^{i}\left(1+\frac{\Delta_{+}}{5}\right)=T_{+}(h+),
$$

where $\Delta_{+}=\frac{v_{+}^{2}}{3 \alpha_{+}+\kappa_{+}}$, while stripes are stable in the range $T<T_{+}^{i}\left(1-4 \Delta_{+}\right)=T_{+}(s)$. As a result, hexagons and stripes 
are simultaneously stable in the bistability domain defined by $T_{+}(h-)=T_{+}^{i}\left(1-16 \Delta_{+}\right)<T<T_{+}^{i}\left(1-4 \Delta_{+}\right)=T_{+}(s)$.

\section{Pattern selection}

Pattern selection resulting from the analysis performed so far in Sec. IV B may then be summarized as follows.

(i) If $\frac{\beta}{\alpha}>\frac{1}{4}$ the only possible steady state is zero and no deposited layer forms since evaporation dominates deposition.

(ii) If $\frac{\beta}{\alpha}<\frac{1}{4}$ there are three possible uniform steady states $c_{0}=0$ and $c_{ \pm}=\frac{1 \pm \sqrt{1-4 \frac{\beta}{\alpha}}}{2}$.

(a) For $T>T_{+}^{i}, c_{0}$ and $c_{+}$are stable and $c_{-}$is unstable. In this bistability regime, localized structures corresponding to isolated dots on the substrate are possible.

(b) For $T<T_{+}^{i}, c_{0}$ remains stable and $c_{-}$unstable; $c_{+}$becomes unstable versus spatial patterns corresponding to stripes or hexagons. Further, $\pi$ hexagons may appear subcritically for $T<T_{+}(h+)$ and are stable for $T_{+}(h-)$ $<T<T_{+}(h+)$. Stripes, in contrast, are stable for $T<T_{+}(s)$. Hence hexagons, stripes, and the uncovered substrate may be simultaneously stable and this can lead to several types of coexisting or localized patterns. Among them are isolated dots or patterned islands coexisting with uncovered domains of the substrate or coexisting domains of hexagonal or striped patterns. Such types of patterns appear frequently in other systems described by reaction-diffusion dynamics or generalized Swift-Hohenberg equations and are analyzed, for example, in [53-59]. These results are illustrated in the schematic bifurcation diagram presented in Fig. 2.

We have thus found that reactive autocatalytic terms in precursor decomposition favor the formation of $H_{-}$hexagonal patterns in the growing layer (similar to nanomesh structures)at temperatures below instability, which is reminiscent of a Frank-Van der Merwe growth with nanostructures. At temperatures above instability, however, localized island formation, which should initiate a Volmer-Weber type of film growth, can occur.

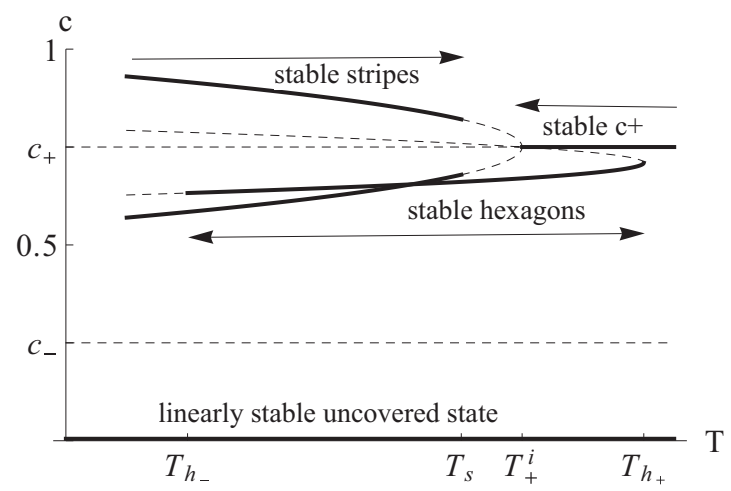

FIG. 2. Schematic bifurcation diagram associated with Eq. (31) in the coverage-temperature plane. Heavy lines represent stable steady states and dotted lines represent unstable ones. According to the temperature, one may observe different multistability domains, which should allow the existence of various types of localized structures.

\section{Weakly nonlinear analysis with $D_{\text {diff }}=D \exp -(\Omega / T)$ and $m>r$}

As already discussed in [26], for diffusion induced by thermally activated atomic jumps, the diffusion coefficient behaves as $D_{\text {diff }}=D \exp -(\Omega / T)$, where $\Omega$ is proportional to the activation energy. This temperature dependence may be discarded for $T \gg \Omega$. However, for decreasing temperatures below instability, one may reach the regime where $T<\Omega$ and diffusion coefficients strongly decrease with decreasing temperature. In this regime relaxation finally dominates diffusion, which rules out any instability at sufficiently low temperatures. There should thus be a finite-temperature range, with an upper and lower bound, for pattern forming instability. A weakly nonlinear analysis will now be performed in this case, in considering diffusion coefficients of the form $D_{\text {diff }}=D c(1-c) \exp -(\Omega / T)$. Equation (31) then becomes

$$
\begin{aligned}
\partial_{t} \sigma(\mathbf{r}, t)= & -\left[\alpha c_{+}\left(2 c_{+}-1\right)+\hat{D}_{+} e^{-\Omega / T}\left(1-\frac{T}{\hat{T}_{+}}+l^{2} \nabla^{2}\right) \nabla^{2}\right] \sigma(\mathbf{r}, t) \\
& -\alpha\left(3 c_{+}-1\right) \sigma^{2}-\frac{\hat{D}_{+} e^{-\Omega / T}\left(1-2 c_{+}\right)}{c_{+}\left(1-c_{+}\right)}\left[1+l^{2} \nabla^{2}\right] \nabla[\sigma(\mathbf{r}, t) . \nabla \sigma(\mathbf{r}, t)] \\
& \left.-\alpha \sigma^{3}+\frac{\hat{D}_{+} e^{-\Omega / T}}{c_{+}\left(1-c_{+}\right)}\left[1+l^{2} \nabla^{2}\right] \nabla\left[\sigma^{2}(\mathbf{r}, t)\right] \cdot \nabla \sigma(\mathbf{r}, t)\right] .
\end{aligned}
$$

Since the maximum of the linear growth rate corresponds to wave vectors such that $q^{2}=q_{m}^{2}=\frac{\hat{T}_{+}-T}{2 l^{2} \hat{T}_{+}}$, instability occurs for

$$
\begin{aligned}
\Sigma_{+} & =\left(1-\frac{T}{\hat{T}_{+}}\right)^{2} \frac{\hat{T}_{+}}{T} \exp -(\Omega / T)>\frac{4 l^{2} \alpha c_{+}\left(2 c_{+}-1\right)}{D} \\
& =\Sigma_{-} .
\end{aligned}
$$

This condition is satisfied for

$$
\begin{aligned}
\frac{4 l^{2} \alpha c_{+}\left(2 c_{+}-1\right)}{D} & <\max \left[\left(1-\frac{T}{\hat{T}_{+}}\right)^{2} \frac{\hat{T}_{+}}{T} \exp -(\Omega / T)\right] \\
& =\left(1-\frac{T^{*}}{\hat{T}_{+}}\right)^{2} \frac{\hat{T}_{+}}{T^{*}} \exp -\left(\Omega / T^{*}\right)
\end{aligned}
$$


where

$$
T^{*}=\frac{\Omega+\hat{T}_{+}}{2}\left[\sqrt{1+\frac{4 \hat{T}_{+} \Omega}{\left(\Omega+\hat{T}_{+}\right)^{2}}}-1\right]
$$

[for small $\Omega, T^{*} \simeq \Omega$, the necessary condition for instability is $\left.4 l^{2} \alpha c_{+}\left(2 c_{+}-1\right) \Omega e \leqslant D\right]$. As mentioned earlier, when the instability condition (37) is satisfied, uniform layers are unstable in a finite-temperature range, with an upper and lower bound. This can be illustrated explicitly for $\Sigma_{-} \lesssim \Sigma_{+}$. In this case, the linear growth rate of (35) may be approximated by

$$
\begin{aligned}
-\alpha c_{+}\left(2 c_{+}-1\right) & +\Phi^{*} \frac{\left(\hat{T}_{+}-T^{*}\right)^{2}}{\hat{T}_{+} T^{*}}-\Phi^{*}\left(\frac{T^{*}-T}{T^{*}}\right)^{2} \\
& =-\Phi^{*}\left[\left(\frac{T^{*}-T}{T^{*}}\right)^{2}-\delta^{2}\right]
\end{aligned}
$$

where $\Phi^{*}=\frac{D e^{-\Omega / T^{*}}}{4 l^{2}}$,

$$
\delta^{2}=\frac{\left(\hat{T}_{+}-T^{*}\right)^{2}}{\hat{T}_{+} T^{*}}-\frac{4 l^{2} \alpha c_{+}\left(2 c_{+}-1\right)}{D} e^{\Omega / T^{*}},
$$

and instability occurs in the temperature range defined by $T_{i-}=T^{*}(1-\delta)<T<T^{*}(1+\delta)=T_{i+}$.

Close to instability, the dynamics is by governed by the evolution of the modes with maximum growth rate, i.e., such that $|\mathbf{q}|=q_{m}$. This is best illustrated in a Fourier transform, where Eq. (35), restricted to these modes, may then be rewritten

$$
\begin{aligned}
\tau^{*} \partial_{t} \sigma(\mathbf{q}, t)= & \frac{\left(T_{i+}-T\right)\left(T-T_{i-}\right)}{\left(T^{*}\right)^{2}} \sigma(\mathbf{q}, t) \\
& +\int_{|\mathbf{k}|=q_{m}} d \mathbf{k}\left[v^{*}\left(q_{m}, k\right)\right] \sigma(\mathbf{q}-\mathbf{k}, t) \sigma(\mathbf{k}, t) \\
& -\int_{|\mathbf{k}|=q_{m}} d \mathbf{k} \int_{\left|\mathbf{k}_{1}\right|=q_{m}} d \mathbf{k}_{1}\left[\kappa^{*}\left(q, k_{1}\right)\right] \sigma(\mathbf{q}-\mathbf{k}, t) \\
& \times \sigma\left(\mathbf{k}-\mathbf{k}_{\mathbf{1}}, t\right) \sigma\left(\mathbf{k}_{1}, t\right) \quad\left(|\mathbf{q}|=q_{m}\right),
\end{aligned}
$$

where $\tau^{*}=\frac{1}{\Phi^{*}}$,

$$
v^{*}(q, k)=\frac{\alpha\left(1-3 c_{+}\right)}{\Phi^{*}}+\left(\frac{\hat{T}_{+}+T^{*}}{\hat{T}_{+}-T^{*}}\right) \frac{1-2 c_{+}}{c_{+}\left(1-c_{+}\right)} \frac{\mathbf{q} \cdot \mathbf{k}}{q_{m}^{2}},
$$

and

$$
\kappa^{*}\left(q, k_{1}\right)=\frac{\alpha}{\Phi^{*}}+\left(\frac{\hat{T}_{+}+T^{*}}{\hat{T}_{+}-T^{*}}\right) \frac{1}{c_{+}\left(1-c_{+}\right)} \frac{\mathbf{q} \cdot \mathbf{k}_{1}}{q_{m}^{2}} .
$$

\section{Stripes}

In this case, amplitude equation analysis shows that for each $T$ in the range $T_{i-}<T<T_{i+}$ and for $\Omega<T^{*}$, stripes of amplitude

$$
\left|A_{m}\right|^{2}=\frac{\left(T_{i+}-T\right)\left(T-T_{i-}\right)}{\left(T^{*}\right)^{2}} \frac{\left(2 c_{+}-1\right)\left(1-c_{+}\right)}{2-c_{+}}
$$

and wave number $q_{m}^{2}=\frac{\hat{T}_{+}-T}{2 l^{2} \hat{T}_{+}}$are the preferred onedimensional patterns since the coefficient of the cubic coupling term of the stripes' amplitude equation is given by

$$
\frac{12 e \Omega l^{2} \alpha}{D}+\frac{1}{c_{+}\left(1-c_{+}\right)} \simeq \frac{2-c_{+}}{\left(2 c_{+}-1\right) c_{+}\left(1-c_{+}\right)} .
$$

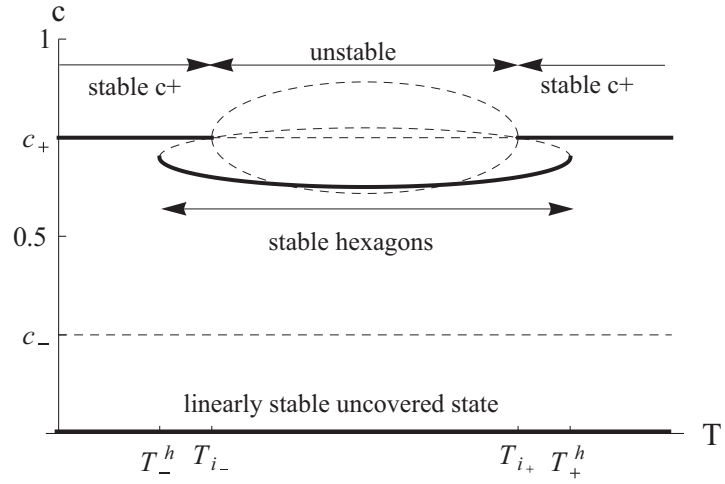

FIG. 3. Schematic bifurcation diagram associated with Eq. (35) in the coverage-temperature plane for $\delta^{2}<\frac{4 c_{+}\left(1-c_{+}\right)}{\left(2-c_{+}\right)\left(2 c_{+}-1\right)}$. Heavy lines represent stable steady states and dotted lines represent unstable ones. According to the temperature, the uniform deposited layer solution, self-assembled hexagonal patterns, and uncovered substrate may be simultaneously stable.

\section{Hexagons}

In the same conditions, the coefficient of quadratic nonlinearities in the amplitude equations for hexagonal patterns is given by

$$
\nu^{*}\left(q_{m}^{2}\right) \simeq \frac{4 e \Omega l^{2} \alpha\left(1-3 c_{+}\right)}{D}+\frac{1-2 c_{+}}{c_{+}\left(1-c_{+}\right)} \simeq \frac{1}{1-2 c_{+}} .
$$

Since it is always negative, an analysis similar to the one performed in previous sections leads to the fact that negative or $\pi$ hexagons exist and appear subcritically for

$$
\left(\frac{T^{*}-T}{T^{*}}\right)^{2}<\delta^{2}+\frac{c_{+}\left(1-c_{+}\right)}{5\left(2-c_{+}\right)\left(2 c_{+}-1\right)}=\delta_{h}^{2}
$$

or

$$
T_{-}^{h}=T^{*}\left(1-\delta_{h}\right)<T<T^{*}\left(1+\delta_{h}\right)=T_{+}^{h} .
$$

In contrast, stripes are only stable for

$$
\left(\frac{T^{*}-T}{T^{*}}\right)^{2}<\delta^{2}-\frac{4 c_{+}\left(1-c_{+}\right)}{\left(2-c_{+}\right)\left(2 c_{+}-1\right)}=\delta_{s}^{2}
$$

or

$$
T_{-}^{s}=T^{*}\left(1-\delta_{s}\right)<T<T^{*}\left(1+\delta_{s}\right)=T_{+}^{s} .
$$

It turns out that if $\delta^{2}<\frac{4 c_{+}\left(1-c_{+}\right)}{\left(2-c_{+}\right)\left(2 c_{+}-1\right)}$, i.e., sufficiently close to threshold, stripes are always unstable and hexagons are stable. As a result, for systems with high atomic mobility $\left(\Omega \ll T^{*}\right)$, pattern forming instability may occur in a finite-temperature range and should be excluded at sufficiently low temperatures as illustrated in the schematic bifurcation diagram presented in Fig. 3. In this temperature range, bistability should occur between hexagonally structured deposited layers and uncovered substrate. This could allow the formation of deposited localized islands of hexagonal patterns on the substrate.

\section{DISCUSSION}

Having proposed an instability mechanism for coverage pattern formation in monolayers adsorbed on a substrate via chemical vapor deposition, it is of course essential to check 
whether this mechanism is experimentally possible. The case of $\mathrm{Al}, \mathrm{Cu}, \mathrm{Ti}$, or TiN films deposited via adsorption-desorption mechanisms only have been discussed in [26]. Furthermore, due to the ever-growing number of experimental processes developed in this field and the impossibility of considering every single experimental system, I will just illustrate the results obtained so far with realistic values of experimental parameters. This may then trigger interest in performing a more focused analysis. To do so, let us consider values in the range of the ones that are commonly used in thin-film deposition.

For example, for a species crystallizing in fcc lattices, with a lattice constant $l=5 \AA$, a pair interaction potential $\epsilon \simeq 0.15 \mathrm{eV}$, and deposited on (100) surfaces, with a very small lattice mismatch, $\epsilon_{0} \simeq 0.6 \mathrm{eV}$ and $T_{c} \simeq 1741 \mathrm{~K}$. For a steady state coverage $c_{0}=0.9, \hat{T}_{0} \simeq 627 \mathrm{~K}$. A realistic value for the atomic diffusion coefficient around this temperature is $D_{0} \simeq 10^{-5} \mathrm{~cm}^{2} \mathrm{~s}^{-1}$. As a result, for deposition rates in the range $\Gamma \simeq 10-10^{3} \mathrm{~s}^{-1}$, in systems where $n=m=1$, $r=2, \frac{\hat{T}_{0}-T_{0}^{i}}{\hat{T}_{0}} \simeq 10^{-4}-10^{-3}$ and $q_{i}=\frac{2 \pi}{\lambda_{i}} \simeq 1.4 \times 10^{7}$ to $4.47 \times$ $10^{7} \mathrm{~m}^{-1}$, which corresponds to a critical wavelength in the range $0.4-0.14 \mu \mathrm{m}$. In fact, for such experimental parameters, the critical wavelength behaves as $\lambda_{i} \simeq\left(\frac{l^{2} D_{0}}{\Gamma}\right)^{1 / 4}$ or for $l=4 \AA$ and $\Gamma \simeq 10^{2} \mathrm{~s}^{-1}$ (which is in the range of typical values for deposition rates in electron-beam evaporation, sputtering, and CVD), $\lambda_{i} \simeq 4 \times 10^{-4}\left(D_{0}\right)^{1 / 4} \mathrm{~cm}$ (for $D_{0}$ expressed in $\mathrm{cm}^{2} \mathrm{~s}^{-1}$ ) and ranges from $40 \mathrm{~nm}$ for $D=10^{-8} \mathrm{~cm}^{2} \mathrm{~s}^{-1}$ to $400 \mathrm{~nm}$ for $D=10^{-4} \mathrm{~cm}^{2} \mathrm{~s}^{-1}$. This behavior is illustrated in Fig. 4.

In contrast, close to threshold, $\Delta=\frac{v^{2}}{\kappa} \simeq \frac{\left(1-2 c_{0}\right)^{2}}{c_{0}\left(1-c_{0}\right)}$. For $c_{0}=$ 0.9 and $\Delta \simeq 7$, hexagonal patterns may appear for $T<1.4 T_{0}^{i}$ while striped patterns are always unstable versus hexagonal ones. Hence, for $T<T_{0}^{i}$, hexagonal patterns should be the only stable ones. For $T_{0}^{i}<T<1.4 T_{0}^{i}$, hexagonal patterns and the uniform steady state are simultaneously stable, allowing the formation of localized hexagonal patterns in uniform background. In fact, it may be shown that stable stripes may only exist for $4 \frac{\left(1-2 c_{0}\right)^{2}}{c_{0}\left(1-c_{0}\right)}<1$ or $0.38<c_{0}<0.62$. Similar results are obtained for systems where $m>r$, except that the uncovered steady state remains linearly stable and stable stripes may only exist for $0.5<c_{+}<0.62$.

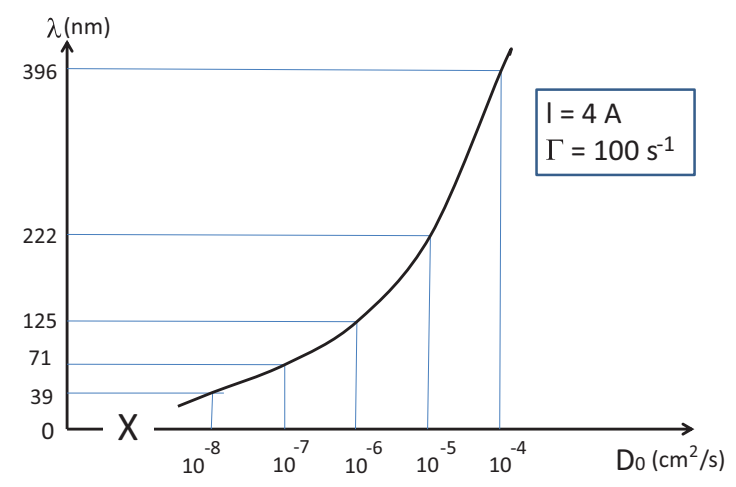

FIG. 4. (Color online) Representation of the critical wavelength dependence on the diffusion coefficient $\lambda_{i} \simeq\left(\frac{l^{2} D}{\Gamma}\right)^{1 / 4}$ for $l=4 \AA$ and $\Gamma=10^{2} \mathrm{~s}^{-1}$ when $D=D_{0} c(1-c)$.

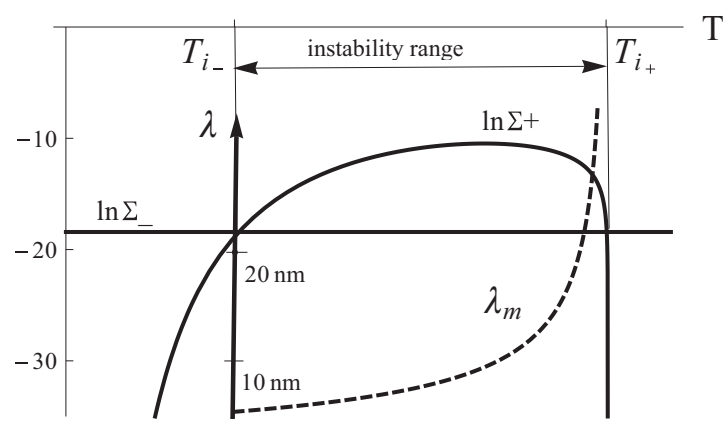

FIG. 5. Schematic representation of the instability condition (36) and the preferred wavelength $\lambda_{m}$ as functions of temperature for $D_{0} \simeq 3 \times 10^{-2} \mathrm{~cm}^{2} \mathrm{~s}^{-1}, \Omega \simeq 3760 \mathrm{~K}, l=5 \AA, \epsilon_{0} \simeq 0.6 \mathrm{eV}, c_{+}=$ 0.9 , and $\alpha=10^{2} \mathrm{~s}^{-1}$.

If one considers thermally activated atomic transport, the diffusion coefficient behaves as $D_{\text {diff }}=D \exp -(\Omega / T)$; realistic values for $D$ and $\Omega$ may be $D \simeq 3 \times 10^{-2} \mathrm{~cm}^{2} \mathrm{~s}^{-1}$ and $\Omega \simeq 3760 \mathrm{~K}$, which correspond to $D_{\text {diff }} \simeq 1.4 \times 10^{-4} \mathrm{~cm}^{2} \mathrm{~s}^{-1}$ at $700 \mathrm{~K}, D_{\text {diff }} \simeq 1.6 \times 10^{-5} \mathrm{~cm}^{2} \mathrm{~s}^{-1}$ at $500 \mathrm{~K}$, or $D_{\text {diff }} \simeq$ $10^{-7} \mathrm{~cm}^{2} \mathrm{~s}^{-1}$ at $300 \mathrm{~K}$. These values are consistent with the ones obtained, for example, through molecular dynamics simulations for surface diffusion coefficient on (100) Al surfaces [4]. For $l=5 \AA, \epsilon_{0} \simeq 0.6 \mathrm{eV}, c_{+}=0.9$, and $\alpha=$ $10^{2} \mathrm{~s}^{-1}$, we obtain $T_{c} \simeq 1741 \mathrm{~K}$ and $\hat{T}_{+} \simeq 627 \mathrm{~K}$. In this case $\left(\Omega / \hat{T}_{+}\right) \simeq 6$ is not small and the instability condition is illustrated in Fig. 5. It turns out that uniform coverage is unstable for $T_{i-} \simeq 147 \mathrm{~K}<T<T_{i+} \simeq 627 \mathrm{~K}\left(1-10^{-4}\right)$. At the lower instability threshold, the preferred wavelength corresponds to $5 \mathrm{~nm}$, while at the upper instability threshold it corresponds to $0.438 \mu \mathrm{m}$. In fact, in the range $300 \mathrm{~K}<T<$ $600 \mathrm{~K}$ it goes from 6 to $20 \mathrm{~nm}$.

\section{CONCLUSION}

In this paper, the evolution of a growing monatomic layer, deposited on a substrate, has been described by a dynamical model of the reaction-diffusion type. This dynamics combines reaction terms and nonlinear diffusion and close to the critical point of the order-disorder transition of the adsorbed layer it corresponds to modified Cahn-Hilliard equations. Up to now, in this approach, the simplest deposition processes were considered. They depend linearly on the atomic coverage of the growing film and may correspond to sputtering or laser assisted deposition. However, in chemical vapor deposition, as discussed here, the reaction part of the dynamics is more complicated and may also be nonlinear for both adsorption and desorption. As a result, when uniform deposited layers become unstable, the competition between nonlinearities arising from reaction or diffusion terms is expected to affect pattern formation, selection, and stability in the deposited layer.

Different types of reaction dynamics have been considered. When the degree of nonlinearity is lower for adsorption than for desorption rates, deposition is dominant at low coverage and favors the initial growth of uniform layers, which may be further destabilized by nonlinear diffusion. In this case, pattern formation phenomena should not qualitatively differ from the case described in [26], where reaction terms are only 
due to linear adsorption and desorption processes. In this case, the effect of more complex reaction processes associated with chemical vapor deposition is only quantitative and modifies the existence and stability ranges of the different patterns, according to the relative importance of reaction- and diffusioninduced nonlinearities. When the degree of nonlinearity is higher for adsorption than for desorption rates, desorption is dominant at low coverage and covered and uncovered domains may be simultaneously stable. According to the temperature, covered domains may become unstable and develop spatial patterns. This may lead to a rich variety of structures that include arrays of patterned or uniform islands or dots in an uncovered background. At low temperatures, a Frank-Van der Merwe type of film growth with nanostructures is recovered. At high temperatures, however, localized island formation, which should initiate a Volmer-Weber type of film growth, can occur. Instability limits and stability domains for the different types of patterns have been derived in the framework of weakly nonlinear analysis. The possible relevance of these results for realistic experimental data has been discussed and promotes interest for further study, either to confirm numerically the proposed pattern formation mechanisms or to link them to experimental phenomena.

\section{ACKNOWLEDGMENTS}

Part of this work was initiated in collaboration with Professor N. M. Ghoniem. An association with the IFISC at the University of the Balearic Islands (Spain) is gratefully acknowledged.
[1] N. Ghoniem and D. Walgraef, Instabilities and SelfOrganization in Materials, Vol. II: Applications in Material Design and Nanotechnology (Oxford University Press, Oxford, 2008).

[2] F. Abraham and G. White, J. Appl. Phys. 41, 1841 (1970).

[3] G. H. Gilmer and P. Bennema, J. Appl. Phys. 43, 1347 (1972).

[4] H. Huang, G. H. Gilmer, and T. Diaz de la Rubia, J. Appl. Phys. 84, 3636 (1998).

[5] T. S. Cale and V. Mahadev, in Thin Films, Vol. 22 (Academic, New York, 1996), p. 175.

[6] H. Gao and W. Nix, Annu. Rev. Mater. Sci. 29, 173 (1999).

[7] N. M. Ghoniem, H. Heinisch, H. Huang, L. Kubin, J. Yu, and S. Yip, eds., Special issue of J. Comput. Aid. Mater. Des. 6(2\&3), 1 (1999).

[8] D. Vvedensky, J. Phys.: Condens. Matter 16, R1537 (2004).

[9] Z. Suo and W. Lu, in Multiscale Deformation and Fracture in Materials and Structures, The James R. Rice 60th Anniversary Volume, edited by T.-J. Chuang and J. Rudnicki (Kluwer Academic, Dordrecht, 2000), pp. 107-122.

[10] Z. Suo and W. Lu, J. Nanopart. Res. 2, 333 (2000).

[11] F. Léonard, M. Laradji, and R. C. Desai, Phys. Rev. B 55, 1887 (1997).

[12] F. Léonard and R. C. Desai, Phys. Rev. B 57, 4805 (1998).

[13] A. A. A. Golovin, S. S. H. Davis, and A. Nepomnyashchy, Phys. Rev. E 59, 803 (1999).

[14] T. V. Savina, P. W. Voorhees, and S. H. Davis, J. Appl. Phys. 96, 3127 (2004).

[15] T. V. Savina, A. A. Golovin, S. H. Davis, A. A. Nepomnyashchy, and P. W. Voorhees, Phys. Rev. E 67, 021606 (2003).

[16] A. A. Golovin, M. S. Levine, T. V. Savina, and S. H. Davis, Phys. Rev. B 70, 235342 (2004).

[17] M. S. Levine, A. A. Golovin, S. H. Davis, and P. W. Voorhees, Phys. Rev. B 75, 205312 (2007).

[18] U. Thiele, J. Phys.: Condens. Matter 22, 084019 (2010).

[19] G. Dewel, S. Métens, M. F. Hilali, P. Borckmans, and C. B. Price, Phys. Rev. Lett. 74, 4647 (1995).

[20] M. Hildebrand, A. S. Mikhailov, and G. Ertl, Phys. Rev. E 58, 5483 (1998).

[21] A. Baski, S. Erwin, and J. Whitman, Surf. Sci. 423, L265 (1999).

[22] H. Ernst, J. Phys. IV 7, C6-55 (1997).
[23] K. Kern, H. Niehus, A. Schatz, P. Zeppenfeld, J. Goerge, and G. Comsa, Phys. Rev. Lett. 67, 855 (1991)

[24] K. Pohl, M. Bartelt, J. de la Figuera, N. Bartelt, J. Hrbek, and R. Hwang, Nature (London) 397, 238 (1999).

[25] D. Walgraef, Physica E 15, 33 (2002).

[26] D. Walgraef, Philos. Mag. A 83, 3829 (2003).

[27] D. Walgraef, Physica E 18, 393 (2003).

[28] G. Abrasonis and K. Morawetz, Phys. Rev. B 86, 085452 (2012).

[29] Q. Hu, N. M. Ghoniem, and D. Walgraef, Phys. Rev. B 75, 075405 (2007).

[30] H. J. Kim, Z. M. Zhao, and Y. H. Xie, Phys. Rev. B 68, 205312 (2003).

[31] H. Kim, Z. Zhao, J. Liu, V. Ozolins, J. Chang, and Y. Xie, J. Appl. Phys. 95, 6065 (2004).

[32] M. Clerc, E. Tirapegui, and M. Trejo, Eur. Phys. J. Spec. Top. 146, 407 (2007).

[33] J. Creighton and P. Ho, Chemical Vapor Deposition (ASM International, Materials Park, OH, 2001).

[34] K. Choy, Prog. Mater. Sci. 48, 57 (2003).

[35] W. Meng, J. Sell, T. Perry, L. Rehn, and P. Baldo, J. Appl. Phys. 75, 3446 (1994).

[36] B. Demczyk, R. Naik, G. Auner, C. Kota, and U. U. Rao, J. Appl. Phys. 75, 1956 (1994).

[37] R. Vispute, R. Chowdhury, P. Tiwari, and J. Narayan, Appl. Phys. Lett. 65, 2565 (1994).

[38] C. Jin, R. Narayan, A. Tiwari, H. Zhou, A. Kvit, and J. Narayan, Mat. Sci. Eng. B 117, 348 (2005).

[39] D. Walgraef, in Proceedings of the 2nd International Conference on Multiscale Materials Modeling, edited by N. Ghoniem (UCLA Publications, Los Angeles, 2004), p. 604.

[40] D. Walgraef, arXiv:1307.5214.

[41] H. S. Fogler, Elements of Chemical Reaction Engineering, 4th ed. (Prentice Hall, Upper Saddle River, NJ, 1999).

[42] Y. Chae and H. Komiyama, J. Appl. Phys. 90, 3610 (2001).

[43] Y. Kajikawa and S. Noda, Appl. Surf. Sci. 245, 281 (2005).

[44] S. De Groot and P. Mazur, Non-Equilibrium Thermodynamics (Dover, New York, 1984).

[45] C. Vidal, G. Dewel, and P. Borckmans, Au-Delà de l'Équilibre (Hermann, Paris, 1994).

[46] A. Newell, Lectures in Applied Mathematics (American Mathematical Society, Providence, RI, 1974), Vol. 15. 
[47] M. Cross and P. C. Hohenberg, Rev. Mod. Phys. 65, 852 (1993)

[48] R. Hoyle, Pattern Formation: An Introduction to Methods (Cambridge University Press, Cambridge, 2006).

[49] N. Ghoniem and D. Walgraef, Instabilities and SelfOrganization in Materials, Vol.I: Fundamentals of Nanoscience (Oxford University Press, Oxford, 2008).

[50] D. Walgraef, Spatio-Temporal Pattern Formation (with Examples in Physics, Chemistry and Materials Science) (Springer, New York, 1996).

[51] M. Bestehorn and H. Haken, Z. Phys. B 57, 329 (1984).

[52] Y. Pomeau, Physica D 23, 3 (1986).

[53] P. Coullet, Int. J. Bif. Chaos 12, 2445 (2002).
[54] J. Burke and E. Knobloch, Phys. Rev. E 73, 056211 (2006).

[55] M. Tlidi, P. Mandel, and R. Lefever, Phys. Rev. Lett. 73, 640 (1994).

[56] U. Bortolozzo, M. G. Clerc, C. Falcon, S. Residori, and R. Rojas, Phys. Rev. Lett. 96, 214501 (2006).

[57] H. Herrero, C. Pérez García, and M. Bestehorn, Chaos 4, 15 (1994).

[58] D. Lloyd, B. Sanstede, D. Avitabile, and A. Champneys, SIAM J. Appl. Dyn. Syst. 7, 1049 (2008).

[59] Localized States in Physics: Solitons and Patterns, edited by O. Descalzi, M. Clerc, S. Residori, and G. Assanto (Springer, Dordrecht, 2010). 